\title{
Overview of the ITER EC Upper Launcher
}

\author{
M.A. Henderson ${ }^{1}$, R. Heidinger ${ }^{2}$, D. Strauss ${ }^{2}$, R. Bertizzolo ${ }^{1}$, A. Bruschi ${ }^{3}$, R. \\ Chavan $^{1}$, E. Ciattaglia ${ }^{4}$, S. Cirant ${ }^{3}$, A. Collazos ${ }^{1}$, I. Danilov ${ }^{2}$, F. Dolizy ${ }^{1}$, J. \\ Duron $^{1}$, D. Farina ${ }^{3}$, U. Fischer ${ }^{2}$, G. Gantenbein ${ }^{2}$, G. Hailfinger ${ }^{2}$, W. Kasparek ${ }^{6}$ \\ K. Kleefeldtt ${ }^{2}$, J.-D. Landis' ${ }^{1}$, A. Meier ${ }^{2}$, A. Moro ${ }^{3}$, P. Platania ${ }^{3}$, B. Plaum ${ }^{6}$, \\ E. Poli ${ }^{5}$, G. Ramponi ${ }^{3}$, G. Saibene ${ }^{4}$, F. Sanchez ${ }^{1}$, O. Sauter ${ }^{1}$, A. Serikov ${ }^{2}$, H. \\ Shidara ${ }^{1}$, C. Sozzi ${ }^{3}$, P. Spaeh ${ }^{2}$, V.S. Udintsev ${ }^{1}$, H. Zohm ${ }^{5}$, C. Zucca ${ }^{1}$ \\ 1 Centre de Recherches en Physique des Plasmas, CRPP - EPFL, CH- \\ 1015 Lausanne, Switzerland \\ 2 Forschungszentrum Karlsruhe, FZK, D-76021 Karlsruhe, Germany \\ 3 Istituto di Fisica del Plasma, ENEA-CNR, 20125 Milano, Italy; \\ ${ }^{4}$ European Fusion Development Agreement - CSU, D-85748, Garching, Germany \\ ${ }^{5}$ Max Planck-Institute für Plasmaphysik, IPP, D-85748 Garching, Germany \\ ${ }^{6}$ Institut fr Plasmaphysik, IPP, D-70569 Stuttgart, Germany;
}

\begin{abstract}
The ITER electron cyclotron (EC) upper port antenna (or launcher) is nearing completion of the detailed design stage and will soon be starting the final build to print design. The main objective of this launcher is to drive current locally to stabilise the NTMs (depositing ECCD inside of the island that forms on either the $\mathrm{q}=3 / 2$ or 2 rational magnetic flux surfaces) and control the sawtooth instability (deposit ECCD near the $\mathrm{q}=1$ surface). The launcher should be capable of steering the focused beam deposition location to the resonant flux surface over the range in which the $q=1,3 / 2$ and 2 surfaces are expected to be found, for the various plasma equilibria susceptible to the onset of NTMs and sawteeth. The aim of this paper is to provide the design status of the principle components that make up the launcher: port plug, mm-wave system and shield block components. The port plug represents the chamber that provides a rigid support structure that houses the mm-wave and shield blocks. The mm-wave system is comprised of the components used to guide the RF beams through the port plug structure and refocus the beams far into the plasma. The shield block components are used to attenuate the nuclear radiation from the burning plasma, protecting the fragile in-port components and reducing the neutron streaming through the port assembly. The design of these three subsystems is described, in addition, the relevant thermo-mechanical and electro-magnetic analysis are reviewed for the critical design issues.
\end{abstract}




\section{M.A. Henderson et al}

\section{Introduction}

The ITER electron cyclotron (EC) system will consist of 20MW (delivered power) continuous wave (CW) at $170 \mathrm{GHz}$ (first harmonic ordinary mode) for heating and current drive (H\&CD) applications, which will be injected into the plasma via either an equatorial (EL) or upper (UL) antenna (or launcher). An in-line switch installed in the transmission line (TL) is used to divert the power to either launcher depending on the physics requirements. For example, if the EC system is to be used for controlling magneto-hydrodynamic (MHD) instabilities the power is directed to the four UL $[1,2]$ providing access to the outer half of the plasma. If central central heating and current drive is required the power is directed to the EL $[3,4]$ providing access to the inner half of the plasma. This paper concentrates on the development work associated with the upper launcher, the readers are encouraged to see references $[3,4]$ for information on the EL.

The UL will occupy four ports and be used for the control of MHD instabilities such as stabilizing the neoclassical tearing mode (NTM) [5] or the sawtooth oscillation [6], which require a very localized and peaked current density profile $\left(j_{C D}\right)$. The physics for NTM stabilization requires the upper launcher to deposit the $j_{C D}$ within the magnetic island associated with the NTM, which is expected to be fully stabilized if the peak in $j_{C D}$ exceeds that of the local $j_{B S}$ by a factor of 1.2 (or $\eta_{N T M} \geq 1.2$ ) based on a fit of multi-machine database [7]. In addition, the launcher has to steer the beam over a relatively wide range across the plasma cross section where the relative flux surfaces susceptible to NTMs are expected to occur. The scanning range was determined by the location of the $\mathrm{q}=2$ and $3 / 2$ flux surfaces for the ITER scenarios 2, 3a and 5 at a variety of normalized beta $\left(\beta_{N}\right)$ and plasma inductances $\left(l_{i}\right)$ to provide an estimated range in which the NTMs could be found on ITER, which resulted in an access range covering $0.5 \leq \rho_{T} \leq 0.86[8]$ (where $\rho_{T}$ is the square root of the toroidal flux), as shown in figure 1 . Note that the condition of $j_{C D} / j_{B S} \geq 1.2$ must be achieved over the entire scanning region with the available EC power. The optical configuration should focus the beam such that less than the full EC power is required to achieve full stabilisation in the event that some gyrotrons are not available or a greater $j_{B S}$ is realised in ITER than expected.

In order to achieve the narrow deposition profile in the plasma needed for NTM stabilisation, the optical layout of the UL focuses a relatively large beam $w \simeq 60 \mathrm{~mm}$ (radius of beam where the E-field is $e^{-1}$ times the beams peak value) to a small beam waist of $w_{0} \simeq 20 \mathrm{~mm}$ at a distance of $\sim 1.6 \mathrm{~m}$ into the plasma. The optical design has been optimised based on dedicated beam tracing analyses to insure that the $j_{C D}$ is maximised over the entire scanning range $[9,10]$. The resulting calculated $\eta_{N T M}$ exceeds by more than $50 \%$ the targeted value of 1.2 assuming $20 \mathrm{MW}$ of injected power as shown in table 1. Recent improvements to the optical design have increased the scanning range to access into $\rho_{T} \leq 0.3$, while maintaining equivalent NTM stabilisation efficiencies [11]. The increased access range is useful for accessing the $\mathrm{q}=1$ surface, providing control of the sawtooth oscillation by depositing driven current either just inside or outside the $\mathrm{q}=1$ surface for either de-stabilising (shortening the sawtooth period) or stabilising (lengthening the sawtooth period) [6]. The effectiveness of the ECCD to control the sawtooth period depends on how the local shear on the $\mathrm{q}=1$ surface is modified, which has a $j_{C D} / w_{C D}$ dependence [13] $\left(w_{C D}\right.$ corresponds to the deposition width). This is similar to the criteria for NTM stabilisation but with the addition of the $1 / w_{C D}$ dependence, which favors an even narrower deposition width. Originally, the ITER Project Integration Document [14] had access to the q=1 surface achievable only from the EL. The EL steers a beam in the toroidal plane for a maximum driven current near the plasma center $\left(\rho_{T} \leq 0.4\right)$. To access the region where the $\mathrm{q}=1$ surface is expected $\left(0.3 \leq \rho_{T} \leq 0.5\right)$, a large toroidal angle is required so that the deposition location is Doppler shifted further outward. The Doppler shift also broadens the deposition 


\section{Article: Overview of the ITER EC UL}

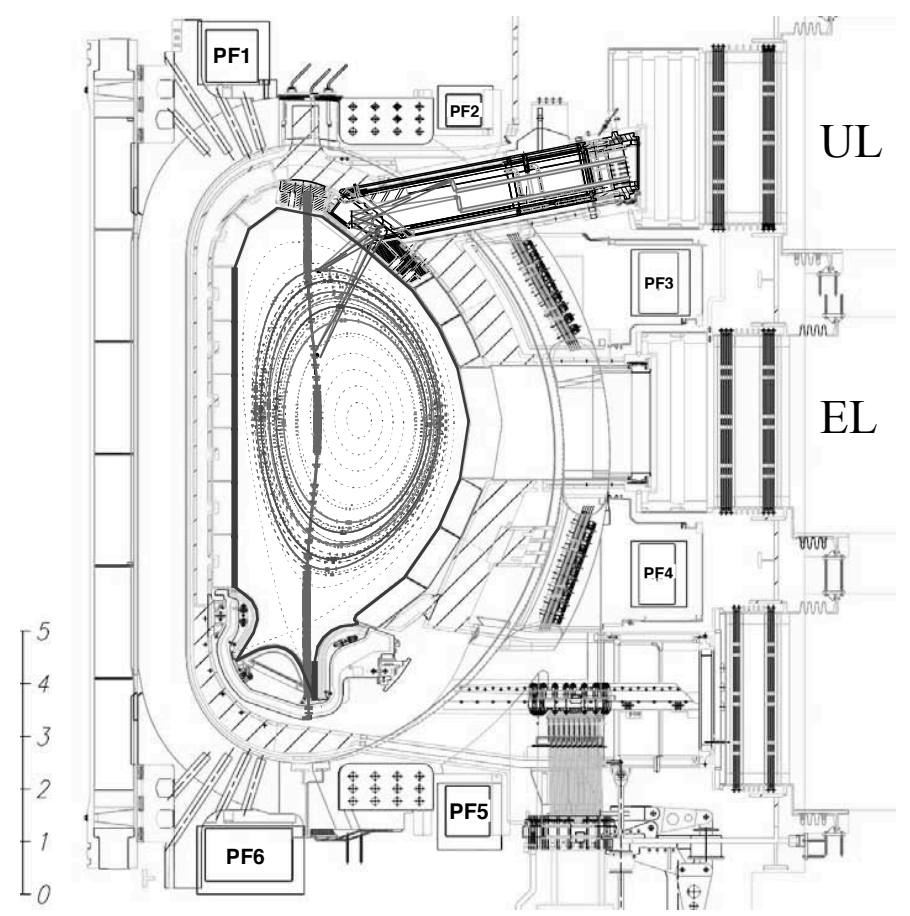

Figure 1. The cross section of ITER with the location of the equatorial (EL) and upper launcher (UL) ports identified. A simplified UL is placed in the upper port with the beams aimed at the inner and outer most expected location of the NTMs.

width, increasing $w_{C D}$ and, in turn, decreasing $j_{C D} / w_{C D}$ such that a significant amount of power is needed to control the sawtooth oscillation $[11,12]$. The narrow and peaked deposition profile obtained from the UL offers a greater control of the sawteeth with less injected power than the EL. For this reason the UL access range was increased to include access to the $\mathrm{q}=1$, which would improve the EC systems control of MHD activity as well as relax the EL steering range requirements. Note that the EL can then be modified for further improved access, reduction of some of the engineering constraints and increased current profile tailoring capabilities as has been outlined in reference [11].

Table 1. The NTM stabilisation efficiency, $\eta_{N T M}=\operatorname{peak}\left(j_{C D}\right) / j_{B S}$ estimated for the upper launcher assuming $20 \mathrm{MW}$ of injected power or the required injected power assuming $\eta_{N T M}=1.2$ is required for complete stabilisation for the various flux surfaces envisioned susceptible to NTMs.

\begin{tabular}{|r|c|c|}
\hline Scenario & $\eta_{N T M}$ & $P_{N T M}[\mathrm{MW}]$ \\
\hline $2(\mathrm{q}=2)$ & 3.53 & 6.8 \\
$2(\mathrm{q}=3 / 2)$ & 2.52 & 9.5 \\
\hline $3 \mathrm{a}(\mathrm{q}=2)$ & 2.69 & 8.9 \\
$3 \mathrm{a}(\mathrm{q}=3 / 2)$ & 1.82 & 13.2 \\
\hline $5(\mathrm{q}=2)$ & 2.07 & 11.6 \\
$5(\mathrm{q}=3 / 2)$ & 1.93 & 12.4 \\
\hline
\end{tabular}

The aim of this paper is to describe the optical and mechanical subsystems that form the launcher, highlighting the technical limitations of these components, which limit the further increased performance of the UL relative to what has been obtained as shown in table 1. An overview of the launcher covering the port plug, optical system and nuclear shielding material is given in chapter 2. Next the analyses of the induced forces during a vertical 


\section{M.A. Henderson et al}

displacement event (VDE) in critical components are described. Then in chapter 4. the thermal mechanical aspects of critical components are presented followed by a summary and conclusion.

\section{Description of Launcher subsystems}

The $\mathrm{R} \& \mathrm{D}$ associated with the UL is nearing completion of the detailed design stage and is progressing toward a build-to-print design of all subsystems, which include the port plug structure, mm-wave components, nuclear shielding elements and the associated auxiliary systems. The design work is following the procurement schedule with the planned delivery and installation of the launcher into the ITER vacuum vessel in the $\sim 2015$ time frame prior to the first plasma planned for March 2016. In order to simplify the design and reduce the overall R\&D costs, the EU design team is seeking out other ITER systems for similarities in design and/or analyses. For example a common port plug design is being considered between the UL and the diagnostic port. Also similarities in the mm-wave system for the UL and the EL are being identified, for example the diamond window design, wave guide components and auxiliary systems. Utilizing common designs avoids duplicating the $\mathrm{R} \& \mathrm{D}$ costs as well as the ITER spare parts requirements and simplifies maintenance procedures.

This chapter is devoted to describing the three main subsystems of the UL: components associated with the mm-wave propagation, port plug structure and the shield blocks to attenuate the nuclear radiation. The three systems are inter related as the port plug defines the available space for the mm-wave system and sufficient space must be reserved for the shield blocks to prevent neutron streaming down the port plug body. The launchers capability to provide a narrow beam deposition at the resonance surface over the entire desired access range depends on the size of the focusing and steering mirrors, the larger the mirrors the more narrow the beam can be focused far into the plasma. The mirror size is limited to the available volume inside the port plug, which is a structure defined by the available space between the poloidal and toroidal field coils. The port plug structure has to be rigid enough to support the optical system, shielding components and its own weight in a simplified enclosure for easy insertion and removal. The volume enclosed by the port plug is then shared between the structure's walls (providing a rigid structure), the optical system and the shielding material to insure low levels of nuclear irradiation to critical components in and near the port plug structure.

\section{1. $\quad$ Port plug}

The port plug [15] is divided into three main sections: blanket shield module (BSM), main structure and the launcher back end, as shown in figure 2. A closure plate separates the launcher back end (available for human access via the port cell) from the launcher main structure (at torus pressure), these sub-components are welded together to form a single unit. The BSM is bolted to this assembly at the plasma end of the structure, note that the bolts provide a simple system for attaching and removal of the BSM in the hot cell for accessing the internal components of the launcher for maintenance or replacement. The front wall panel (FWP) forms the plasma facing component and is capable of withstanding high thermal flux coming from the plasma. The surface of the front wall panel is slightly recessed relative to the neighboring blanket shields to avoid direct contact with the plasma and limiting the thermal loading compared to a standard BSM. The whole assembly is inserted into the port duct at the launcher back end to the torus vacuum vessel and sealed using either bolts (with a doubled seal) or a weld.

The port plug structure has to be a rigid system that can support the weight of the mm-wave components, the shield blocks and its own weight all from the connection to the vacuum vessel at the back end. Note that 


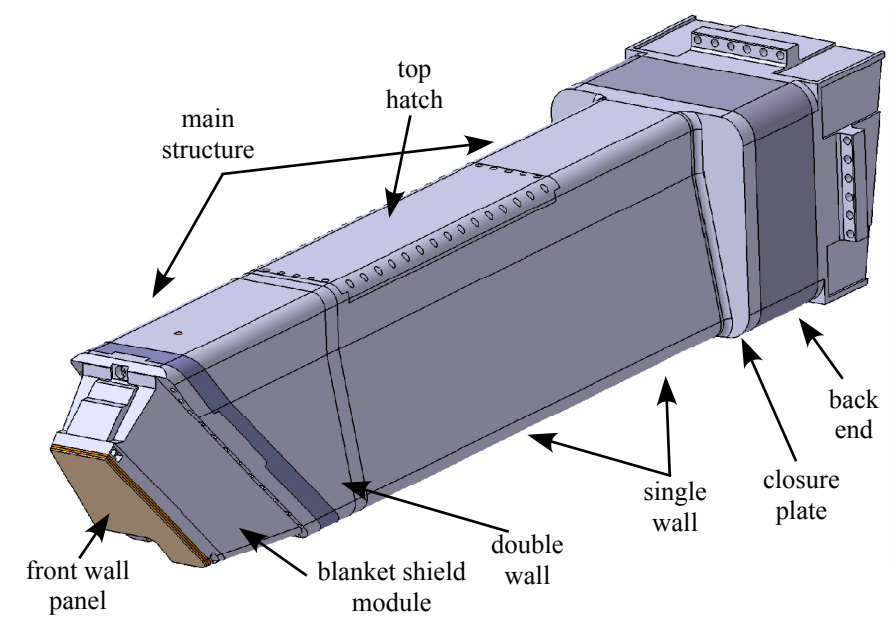

Figure 2. The principle sections of the structural system for the ITER ECH upper port plug.

the whole structure is in a cantilevered configuration, which is free floating from the BSM to the launcher back end. The overall length of the launcher is $\sim 6.1 \mathrm{~m}$, which is mostly the main structure, a tube of $\sim 4 \mathrm{~m}$ in length with a trapezoidal-like structure. The reference design of the port plug structure has a majority of the side walls formed of a doubled wall structure [15] of parallel $30 \mathrm{~mm}$ thick plates as shown in figure 3a. The volume between the two plates is used for free circulation of coolant fed by the blanket water primary heat transfer system (blanket water), which follows a meandering path between the two plates. Apart from offering very efficient and homogeneous cooling system required for evacuating the nuclear heat load generated at the front sections, the double wall sections serve together with the actively cooled internal components as heater elements during bake-out when blanket water is increased to $240^{\circ} \mathrm{C}$ (pressure of $4.4 \mathrm{MPa}$ ) and fed into the main launcher cooling system. Recent design modifications [16-18] to the port plug structure include regions of the side walls with a single (or slim) $55 \mathrm{~mm}$ wall structure in the central region, see figure $3 \mathrm{~b}$. The slim wall offers an equivalent strutctural rigidity, while reducing the manufacturing cost and complexity. In addition the slim wall design aims at a common design with that of the diagnostic port design [19, 20]. A removable hatch is included in this region for increased access to the port centre, which simplifies the access to the mm-wave and shield components or to the diagnostics in the case of the diagnostic port.

The side walls of the BSM (see figure 3c) are formed of a similar double wall structure with internal coolant flow for evacuating the absorbed thermal energy from the plasma or for circulating the heated coolant during baking cycles. The BSM is attached to the main structure using a bolted flange, which provides a simple system for removal of the BSM in the hot cell for axial access to the components at the launcher front end. The FWP is the main plasma facing component of the port plug structure. It is comprised of a multi-layer structure formed as a hot-isostatically pressed (HIPped) compound of a beryllium protection layer (10mm), Cu-alloy sheets $(22$ $\mathrm{mm}$ ) with imbedded stainless steel cooling tubes and a stainless steel back plate $(49 \mathrm{~mm})$ with drilled cooling channels [21]. The panel is welded its outer edge to the BSM housing.

The launcher back end also incorporates a double walled structure (see figure 3d) with free circulating coolant between the walls, which is used to heat the port plug structure during baking cycles. Note that heating the port plug in the front and back regions is sufficient to achieve a relatively uniform temperature of $\sim 240^{\circ} \mathrm{C}$ as required for the torus baking. 


\section{M.A. Henderson et al}

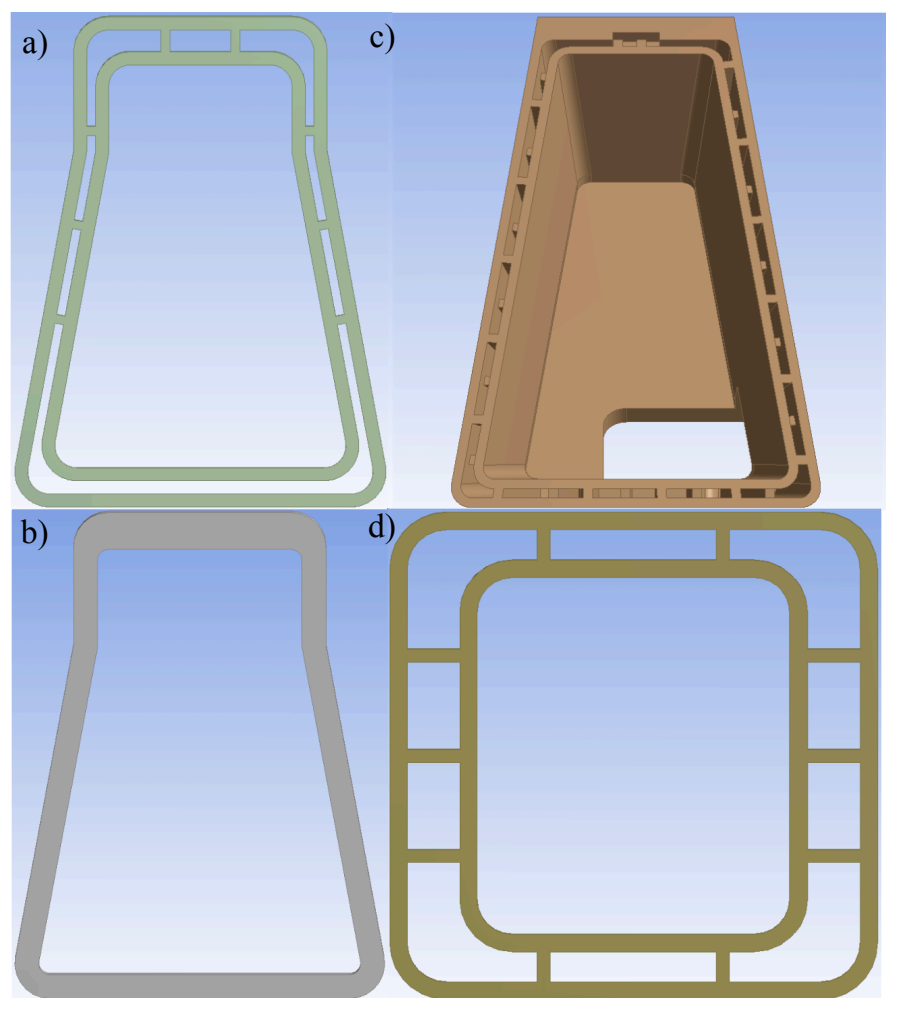

Figure 3. The cross section of the a) double walled trapozoidal, b) single walled trapozoidal, c) BSM region and d) launcher backend of the port plug structure.

\subsection{The mm-wave system}

The layout of the optical components depends strongly on the port plug dimensions and physics objectives. The later is characterised by a scanning range and focusing requirements. The scanning range corresponds to the angular steering of the beam to access the relevant flux surfaces associated with the NTMs (roughly $0.5 \leq \rho_{T} \leq 0.86$ ) and the possible location of the $\mathrm{q}=1$ surface (roughly $0.3 \leq \rho_{T} \leq 0.5$ ). The launcher optics should provide the smallest deposition profile over the entire the scanning region, which depends on the beam spots size in the plasma, launch point, toroidal injection angle, plasma temperature and density profiles. The optimum launch point for the upper port can be determined by drawing lines tangent to the relevant flux surfaces (over the deposition region), as shown in figure 4. A beam trajectory along a tangential line minimises the spread of the beam across multiple flux surfaces due to the Doppler broadening of the beam. The intersection of these lines then represent the optimum launcher point, represented by the red 'X' of figure 4, which occurs in the BSM just below the launcher port plug. 'Dropping' the port plug using the lower BSM increases significantly the NTM stabilisation efficiency and control of the sawtooth oscillation [22]. However, lowering the port plug would incur significant delays in the ordering of the vacuum vessel, resulting in significant delays for the ITER project.

Using the present vacuum vessel geometry implies that the preferred launch point is as close as possible to the optimum ( red 'X' of figure 4), which would be at the base of the BSM as shown in figure 4 by the two circles representing the lower (LSM) and upper (USM) steering mirrors. Once the optimum launch point is fixed, the rest of the optical layout is rather straight forward. A focusing mirror is placed in front and above the steering mirrors, this location minimises the distance from the focusing mirror to the resonance location in the plasma 


\section{Article: Overview of the ITER EC UL}

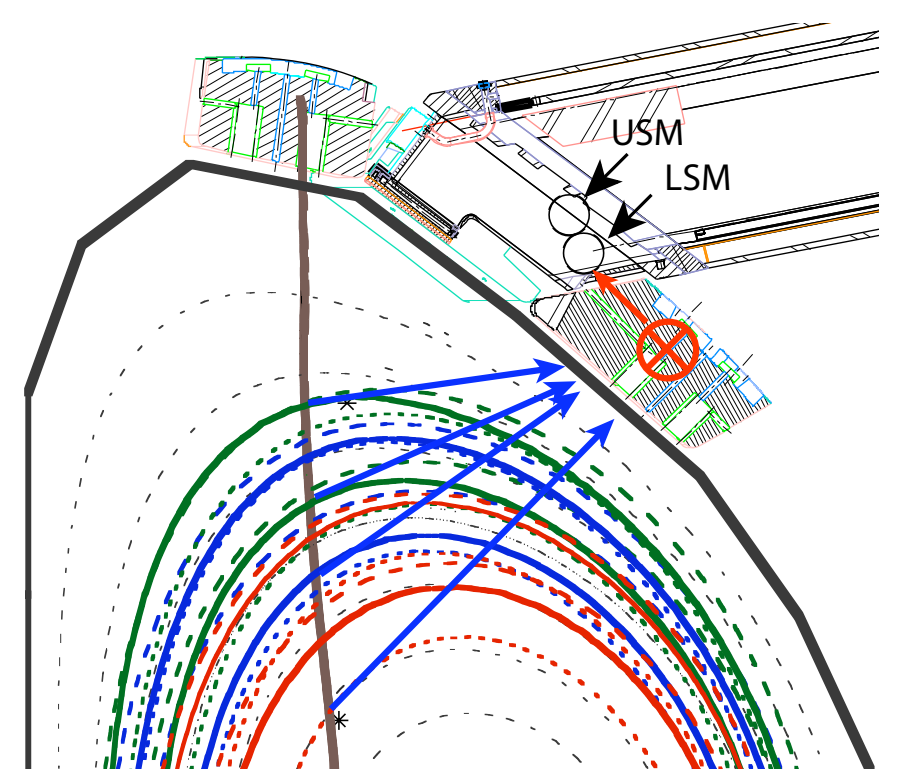

Figure 4. The poloidal view of ITER showing the launcher and plasma flux surfaces. The optimum launch point for a minimum deposition width occurs at the intersection of the tangent lines (red X) from the flux surfaces at the deposition location.

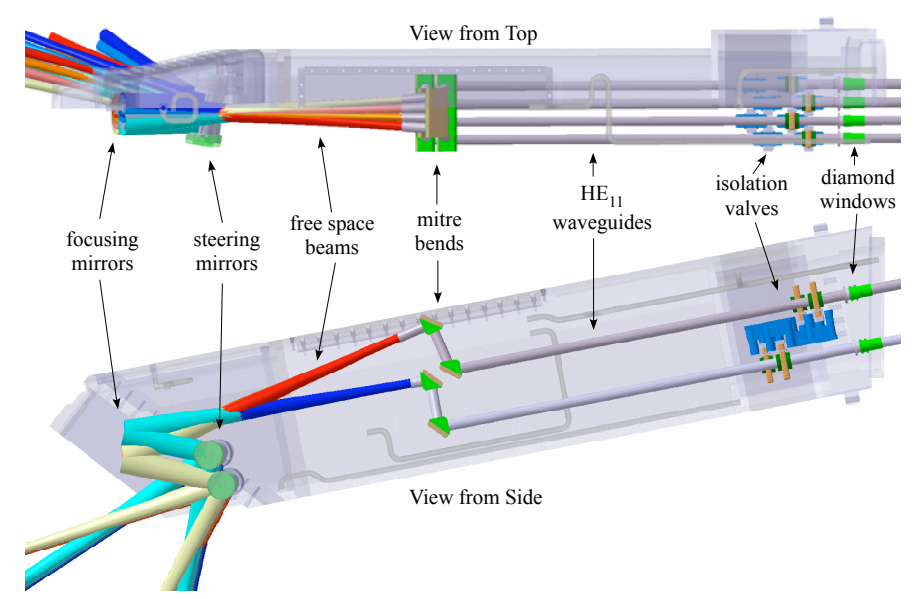

Figure 5. A top and side view of the UL with the principle mm-wave components identified.

and provides adequate space for a relatively large beam spot size on the mirror to focus a small beam waist far into the plasma. The beams incident on the focusing mirror are projected from the wave guide aperture placed behind the steering mirrors. The final configuration is shown in figure 5, where two sets of focusing and steering mirrors are used to send eight beams (each up to 2.0MW) into the plasma. The four beams on each mirror have slightly different launch points, which result in small shifts of the deposition location assuming the same poloidal $(\alpha)$ and toroidal $(\beta)$ launch angles. This is partially corrected by introducing a small toroidal divergence between the four beams, such that the deposition is nearly coincident throughout the scanning region of the plasma $[10,23]$. Note that having the four overlapping beams share one focusing and one steering mirror optimises the available space in the narrow BSM, maximising the beam size for improved focusing (see table 2 for beam parameters).

The toroidal curvature of the focusing mirror is increased to compress the four beams on to the relatively 


\section{M.A. Henderson et al}

small steering mirror ( $290 \mathrm{~mm}$ toroidal by $200 \mathrm{~mm}$ poloidal), but creates an astigmatic beam with a smaller beam waist in the toroidal direction, increasing the beam divergence and spot size in the plasma and decreasing the peak driven current density between 5 to $15 \%$, principally when the USM is directed toward the q=1 surfaces $\left(0.3 \leq \rho_{T} \leq 0.5\right)$. Note that the effect of the astigmatic beams is significant when there is a large distance between the focusing mirror and the absorption region, which is the case when the beams are aimed toward the plasma center for sawtooth control. In the case of the NTM stabilisation $\left(0.5 \leq \rho_{T} \leq 0.86\right)$, the distances are relatively short and there is little difference between a circular or astigmatic beam. The astigmatism can be reduced by replacing the mitre bends with free space mirrors, which provides a greater flexibility in orientating and shaping the beams prior to the focusing mirror. The curvature of the two new mirrors can be chosen to compress the beam assembly and input radius of curvature prior to the focusing mirror to reduce the overall astigmatism for the USM. An example of this improvement is shown in the preliminary optical designs with no mitre bends (no MB case) of table 2 relative to the existing design with mitre bends (with MBs). Note that the no mitre bend case is under development and a CATIA model has not been finalised.

Table 2. Characterization of the injected beams into the plasma for the EP launcher, where $w_{0}$ is the beam waist projected a distance of $d_{0}$ from the focusing mirror. Note that there is about $0.6 \mathrm{~m}$ between the focusing and steering mirrors.

\begin{tabular}{|r|c|c|c|c|}
\hline Steering mirror & $w_{0}$ (Pol.) $[\mathrm{mm}]$ & $d_{0}$ (Pol.) $[\mathrm{m}]$ & $w_{0}$ (Tor.) $[\mathrm{mm}]$ & $d_{0}$ (Tor.) $[\mathrm{m}]$ \\
\hline USM with MBs & 29.0 & 2.7 & 17.4 & 1.8 \\
\hline LSM with MBs & 21.0 & 2.2 & 19.1 & 2.0 \\
\hline \hline USM no MBs & 29.0 & 2.7 & 19.1 & 1.8 \\
\hline LSM no MBs & 21.0 & 2.2 & 20.9 & 2.2 \\
\hline
\end{tabular}

There are other advantages of using free space mirrors rather than the mitre bends and wave guides. Using free space mirrors are less expensive than the mitre bend and waveguide components, thus reducing the overall costs. In addition, the beam spot size on the mirrors can be increased to reduce the peak power density on the mirrors, simplifying the engineering design. Changing to a no mitre bend design may significantly impact the neutron streaming fluence at the closure plate. The beams can be overlapped occupying a smaller volume, increasing the available space for shield components. Also, the beam paths can be orientated to avoid straight

paths through the launcher structure, as is the case for the upper row of waveguides in the vicinity of the mitre bends. The present design has more than adequate shielding with the neutron fluence less than one order of magnitude down from the ITER limits [35]. The full quasi optical design will aim at maintaining or reducing this level.

In 2004, the ITER-IO made available four ports for the UL, which would imply 6 beams per port plug for the 24 gyrotrons to be installed during the first phase of operation. The present UL design is capable of injecting up to 8 beams (each of $2 \mathrm{MW}$ ), thus requiring only three ports. Rather than saving one port, ITER-IO recommended using the fourth port in such a way as to improve the UL mechanical reliability and if possible increase the physics performance. Following this recommendation, the Extended Performance upper launcher (or EP-UL) design was developed. The EP-UL provides access to all of the relevant NTMs and the $\mathrm{q}=1$ surfaces by spreading out the scanning regions of the two steering mirrors as compared to accessing just the NTMs as illustrated in figure 6. This forms three access regions: an inner region $\left(\sim 0.3<\rho_{T} \leq 0.6\right)$ accessible from only the USM with 13.3MW of available power, a mid region $\left(\sim 0.6<\rho_{T} \leq 0.8\right)$ accessible from either the USM and LSM with the full 20MW and an outer region $\left(\sim 0.8<\rho_{T} \leq 0.86\right)$ accessible from the LSM with 13.3MW. The RF power would be switched between the USM and LSM using an in-line switch similar to that used for 


\section{Article: Overview of the ITER EC UL}
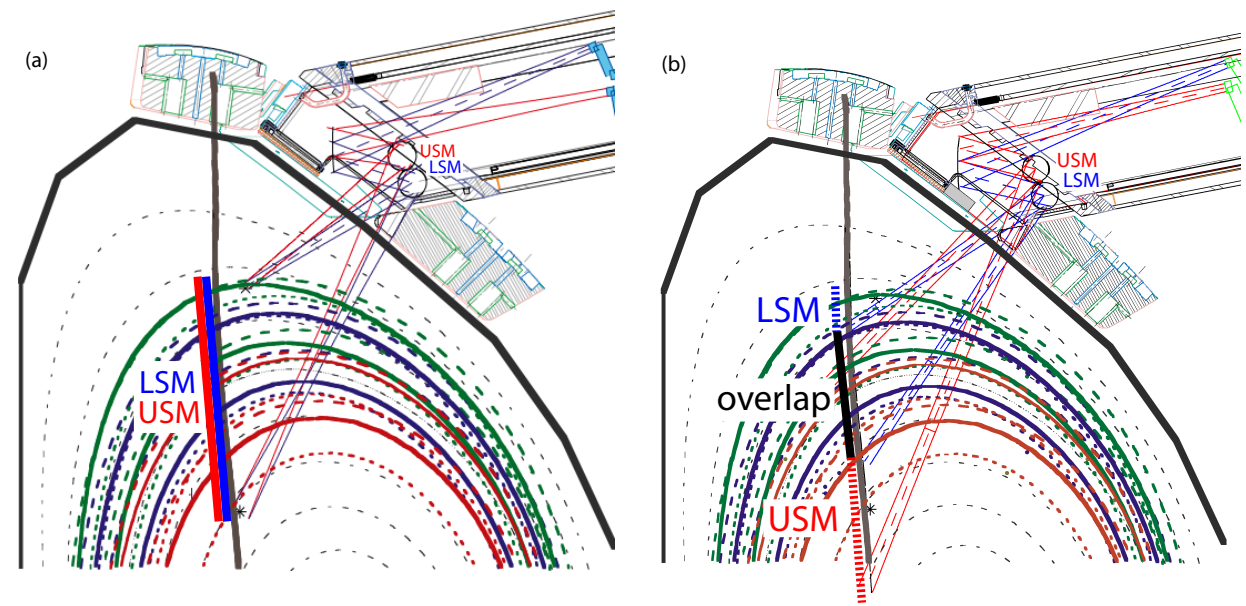

Figure 6. The scanning range of the upper (USM) and lower (LSM) steering mirrors of the a) NTM launcher and b) EP-UL.

switching between the equatorial and upper launchers. The advantages of the EP-UL design include a larger access in the plasma for better physics performance (which also offers relaxing and further optimization of the equatorial launcher) $[24,25]$ and a reduction of the steering mirror rotation relaxing the engineering constraints on the steering mechanism $[26,27]$. The smaller the rotation required of the steering mirror, the lower the stresses induced in the actuator performing the mirror rotation. As the stresses decrease, the number of steering cycles to failure increases, increasing the reliability and longevity of the steering mechanism.

The focusing mirror of the NTM launcher focuses all output beams with a waist of $21 \mathrm{~mm}$, optimised for the steering range covering the envisioned $\mathrm{q}=3 / 2$ and 2 NTMs. The optical design for the EP-UL has been changed such that the output beams for the two steering mirrors are individually optimised for the distances from the mirror to the deposition location. The path length from the focusing mirror to the resonance location via the LSM is roughly $2.2 m$ allowing for a strong focusing equivalent to the NTM design. However, the path length from the focusing mirror to the resonance location via the USM is roughly $2.8 \mathrm{~m}$. The longer path length for the USM favors a larger beam waist $(\sim 29 \mathrm{~mm})$ to avoid a strong beam divergence and a large spot size when aiming toward the plasma centre. Note that the optical description of table 2 corresponds to the EP-UL.

The EP-UL design achieves the relaxation of the engineering constraints, while improving the physic capabilities, which was the objective in utilising the fourth port. Note that the steering mechanism reliability was the main concern associated with adapting the front steering mechanism concept for the upper port, where the majority of critical design issues of the present launcher design were associated with the steering mirror ensemble [24]. In order to minimize the risks associated with mechanical failure, an innovative design of the steering mechanism [26, 27] (see Figure 2a) is being developed that provides rotation of the steering mirror based on the compliant deformation of structural components offering a frictionless and backlash free mechanical movement and avoiding the in vessel tribological difficulties inherent in present day steering mechanisms. Traditional ball bearings are replaced with elastically compliant flexure pivots [28] and the movement is controlled using an integrated gaseous helium pneumatic actuator system working against preloaded compressive springs in place of the push-pull rods. This eliminates the components that typically grip offering an improved reliability and precision in controlling the steering mirror angle. The bellows are pressurized from the outside, which offers a more stable configuration avoiding the squirm instability when the bellows are internally pressurized. A pair of coiled cooling pipes with either a single or double wall provides a flexible coolant feed to the mirror, following 


\section{M.A. Henderson et al}

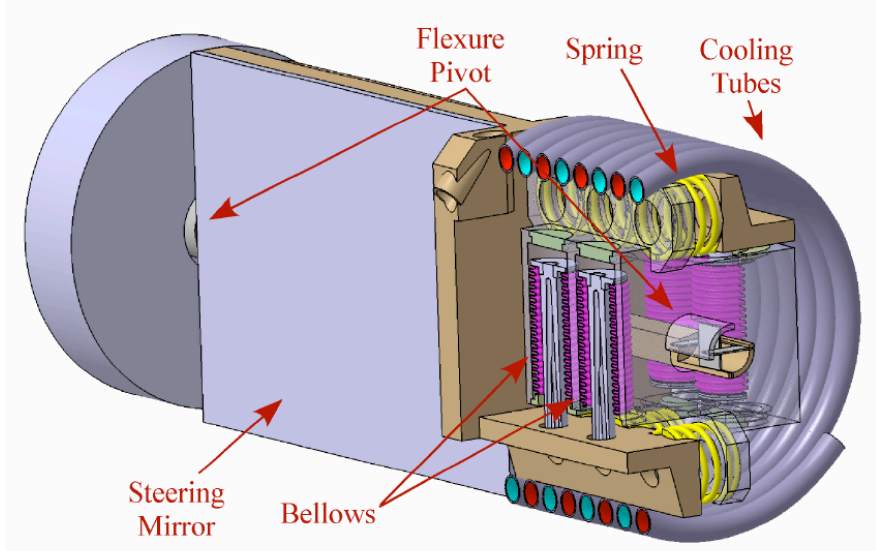

Figure 7. The balanced configuration of the steering mirror ensemble with the steering mirror positioned between the two flexure pivots.

a similar design to that proposed for the EL [3]. The coiled pipes are relatively rigid and are installed with a gap between spirals to avoid contact at any rotation angle.

Efficient stabilisation of the NTMs require all the beams deposition profiles to be coincident in the plasma, which requires a steering acuracy of $\sim 0.1^{\circ}$. To achieve this accuracy, two systems are envisioned to measure the steering mirror angle: the servo valve pressure (located outside the closure plate) and a direct measure on the steering mirror. The servo valve can control the pressure in the bellows with an accuracy of $\pm 5 m b a r$ over a range of $\sim 10$ bar corresponding to an angular accuracy of $\leq \pm 0.02^{\circ}$, which is well within the desired accuracy of $\leq 0.1^{\circ}$. However, ITER-IO requested an active angular measuring system directly on the mirror as a back-up system to ensure knowledge of the beam injection angles and provide a cross calibration of the servo valve system as the springs constants of the spiral cooling tubes, bellows, flexure pivots and springs will change in time. Several candidate systems are under investigation that include variable capacitive plates, electrical contact switches and interferometry. The final choice will depend on the system's reliability and simplicity to install the system into the steering mechanism. Note that even though the four beams leaving a given steering mirror will be accurately controlled via a combination of the two systems mentioned above, the accuracy of depositing all 24 beams coincident in the plasma strongly depends on the diagnostic systems used to distinguish the EC deposition location from each beam sets from the eight steering mirrors [29]. The launchers will also require in-situ calibration that could include sweeping the beams through the plasma and cross calibrating the steering mechanism using well defined plasma 'targets' for example the $\mathrm{q}=1$ surface as was used for the alignment of the TCV EC launchers [30].

The main failure mode envisioned for the UL steering mechanism is fatigue of the bellows used in the pneumatic actuator. Several materials have been investigated for manufacturing the bellows to increase the longevity, while remaining compatible with the ITER environment. The fatigue curves as a function of steering range for two candidate materials (nickel and Inconel) are shown in figure 8, based on the Expansion Joint Manufacturers Associations (EJMA) [31] standards. In order to achieve the access requirements associated with the EP-UL the steering mirror should be rotated $\pm 5.5^{\circ}$ ( $\pm 11^{\circ}$ for the beam). The Inconel bellows can withstand $>100^{\prime} 000$ cycles prior to the onset of cyclic fatigue (taking into account nuclear effects), which is well above the desired $<30^{\prime} 000$ cycles based on estimates made for NTM stabilisation requirements during the full ITER lifetime [24]. Fatigue testing of the bellows under similar stress conditions are being performed by the bellows 


\section{Article: Overview of the ITER EC UL}

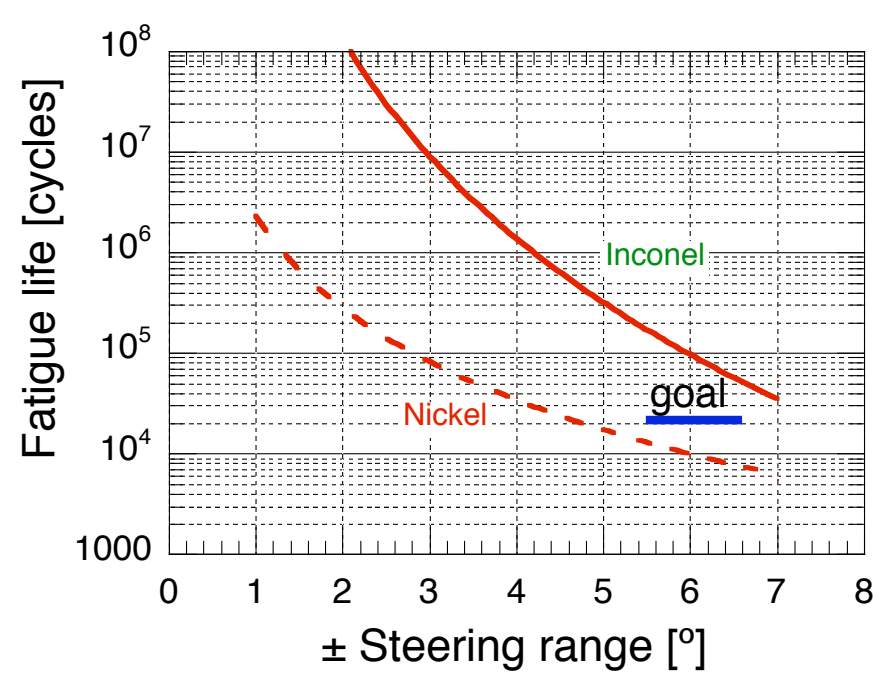

Figure 8. The number of cycles to failure based on EJMA [31] for nickel and Inconel bellows investigated for the UL steering mechansim.

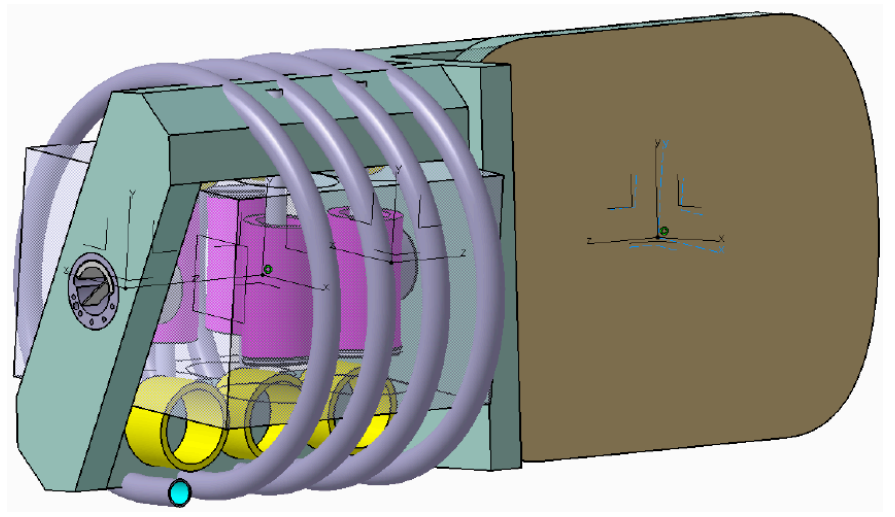

Figure 9. The cantilevered configuration of the steering mirror ensemble with the two flexure pivots incorporated into the steering mechanism.

manufacturer and in a simplified steering mechanism at CRPP to insure compliance with the fatigue curves of figure 8 .

The steering mirror (SM) ensemble of figure 7 is referred to as the balanced SM, which has the SM centered between two flexure pivots. This configuration reduces the overall forces on the flexure pivots during a vertical displacement event (VDE), but requires two supports on either side of the SM and has an increased risk of the halo current flowing through the mirror structure. An alternative configuration is the cantilevered $S M$ as shown in figure 9 , with both flexure pivots incorporated in the steering mechanism. This configuration was the original SM design preposed back in 2003 that was compatible for only two 1MW incident beams.

A comparison of the two steering mechanisms has been performed aiming at an optimum system for the UL. The main advantage of the balanced SM is the reduced forces on the flexure pivots during a VDE. But the design introduces several complications in installing and supporting the system in the port plug. As mentioned above, the balanced mechanism is supported from both sides, which requires a flexible membrane on one side of the assembly to compensate for thermal expansion of the mirror during operation. Also, the flexure pivot and membrane have to be actively cooled requiring a coolant connection on both sides of the steering mechanism 


\section{M.A. Henderson et al}
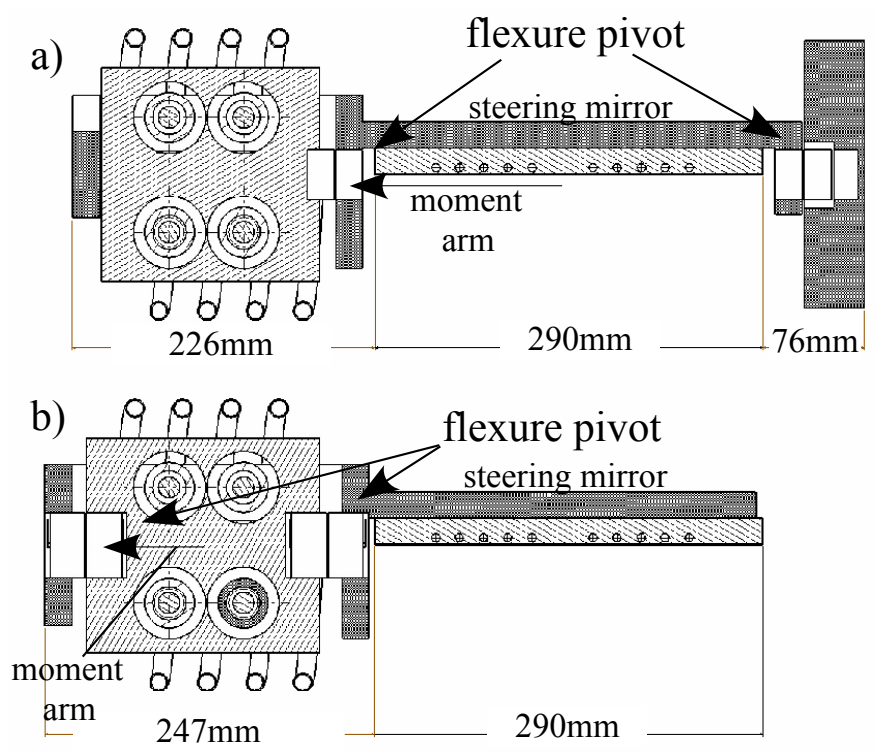

Figure 10. A cut away view of the two steering mechanism configurations: a) balanced and b) cantilevered..

complicating the installation and removal during remote handling operation. The cantilevered configuration avoids these complications by placing the flexure pivot in the steering mechanism body, however, the resultant distances between the flexure pivot is reduced (see figure 10), decreasing the moment arm and increasing the applied forces on the flexure pivot from the induced torque during a VDE (17MA). The design of the flexure pivot has been improved to withstand up to $\sim 3.2 k N$ assuming up to 1000 VDEs. Note that ITER itself is designed to withstand only 100 such VDEs. Optimisation of the steering mirror design has reduced significantly the amount of copper in the mirror edges, which prevents large currents from circulating in the mirror body. As will be described in section 3.2., the forces associated with a VDE from a plasma with 17MA, results in a force of less than $2 \mathrm{kN}$ at the worst rotation angle of the steering mirror.

The advantages associated with the cantilevered configuration has led the design team to adopt this system as the reference design. The manufacturing drawings are now being developed, with the aim to produce and test a prototype system during the 2007-2008 time frame.

An alternative layout of the ex-vessel wave guide components is also under consideration, which increases the maintenance access to the closure plate and critical wave guide components (diamond window and isolation valve). As mentioned above a diamond window [32] is located just outside the closure plate providing a primary tritium barrier between the launcher and transmission line [33]. An isolation valve is placed on the plasma side of the diamond window so that the window can be repaired in-situ without affecting the torus pressure [34]. Both the isolation valve and diamond window fit into the launcher back end as shown in the insert of figure 11, which results in an extremely congested launcher entrance complicating maintenance access to either the isolation valves or the diamond windows. An alternative layout is under consideration that moves both components out of the launcher back end and into the port cell area as shown in figure 11. This modification would simplify the interface [29] between the launcher and transmission line [33] as well as extend the primary tritium barrier an additional $\sim 5 \mathrm{~m}$, requiring the wave guide between the closure plate and diamond window to be of stainless steel and either welded or double sealed connections. In the event the launcher had to be removed for servicing, the intervening wave guide would be removed, the ends capped, while the window and valve would remain in place. 


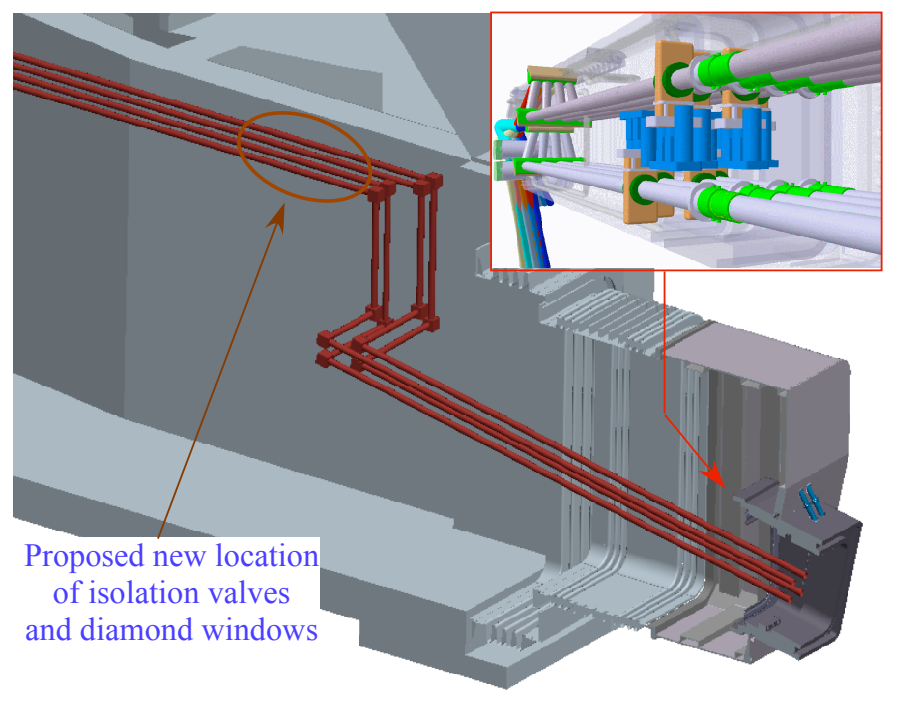

Figure 11. The entrance to the UL with the isolation valves and diamond windows placed prior to the closure plate within the volume of the port plug (see insert) and the alternative location with both placed further from the port plug for improved access to both components and the closure plate.

Note that the diamond window is a part of the primary tritium boundary and therefore will have dedicated diagnostic systems to insure its proper functioning. For example, there will be the possibility to perform in-situ leak testing of the window by leaking in a trace gas between the valve and window and monitoring the presence of the trace gas in the wave guide vacuum pumping section on the gyrotron side of the window [24]. The coolant flow, input and output temperatures will be monitored for estimating the absorbed power. Also, a fibre optic arc detector will view the window and shut down the RF power in the event of an arc. The arc detection system will be either a dedicated system installed on the window housing unit or use an arc detector in a near by mitre bend.

\subsection{Nuclear shield components}

Once the optical system has been specified, the remaining free space is filled with shielding material to reduce the nuclear heating of neighboring components and limit the neutron streaming through the port plug to the port cell. The shield blocks are used to limit the radiation damage in the internal components of the port plug and neighboring material. The radiation damage is calculated using a Monte Carlo code MCNP [36] and assumes the operation of a half power year (estimated integrated time of ITER D-T operation). The analyses associated with the shielding block is not included in the scope of this paper. A review of the analyses is provided in the references [35] and [36].

The shield components have been divided into two regions: main structure and BSM. The shield blocks in the main structure are shown in figure 12. These shields provide the major radiation protection of the launcher components at the back-end and of surrounding structures, for example the vacuum vessel, superconducting coils, and diamond windows. Out of three possible design options (block, tank and modular design), a combination of a modular design has been selected [15] with the aim for further optimisation of the different designs prior to the finalising of the build to print designs. The front shield block is formed by a sandwich of several individual plates, while the back shield is formed by a metal block with machined cooling channels. The space between the two shield blocks are used for the mitre bend dogleg structure. Note that the opening formed in the first 


\section{M.A. Henderson et al}

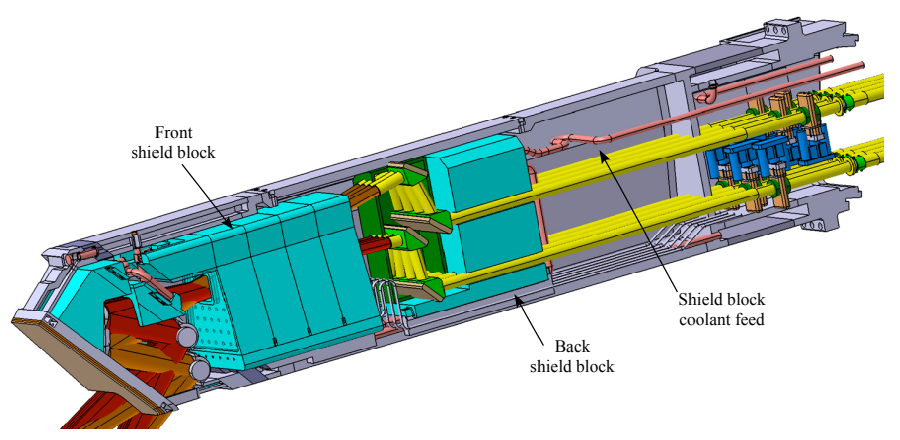

Figure 12. The location of the shield blocks and the piping of the cooling connections in the BSM.

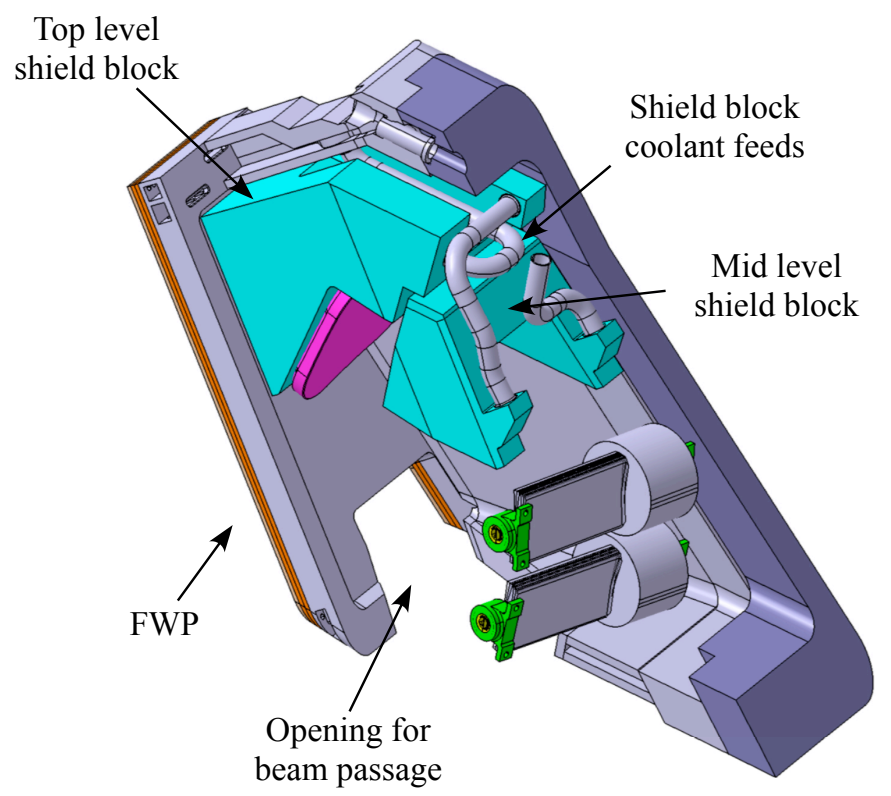

Figure 13. A cut open view of the BSM and the its shield blocks.

shield block for the passage of the free space beam is kept larger than $1.75 \mathrm{w}$ (distance from beam axis to nearest shield surface). The full quasi optical design will increase this distance to $\geq 2 w$ to insure that the side walls do not influence the beam propagation through the shield blocks.

Two shield blocks are also used in the BSM in the top and mid level segments as shown in figure 13. Their cooling lines are integrated into the main launcher cooling system by pipes feeding in the coolant from the BSM housing into the top-level shield block. After the circulation in the mid-level shield block, piping is provided for transfer into the internal shield segments which form the neutron shields in the main frame. In the present configuration the top-level shield block provides fixation and cooling of the focusing mirror, although an alternative design is under investigation that has an independent support of the focusing mirrors that is aligned directly with the steering mirrors to minimise misalignments.

Given the actual geometry of the mm-wave beam configuration, the encased shield block concept is given preference over the potential alternatives, such as the solid shield block used in the main structure of the port plug. The encased shield block concept is particularly suited for large and regularly shaped volumes, the concept is based on filling a welded stainless steel (SS) casing with stacked SS plates and water interspaces which allows an equal distribution of water flow through the shield block as shown in figure 14. Furthermore, by adequate 


\section{Article: Overview of the ITER EC UL}

a)

b)
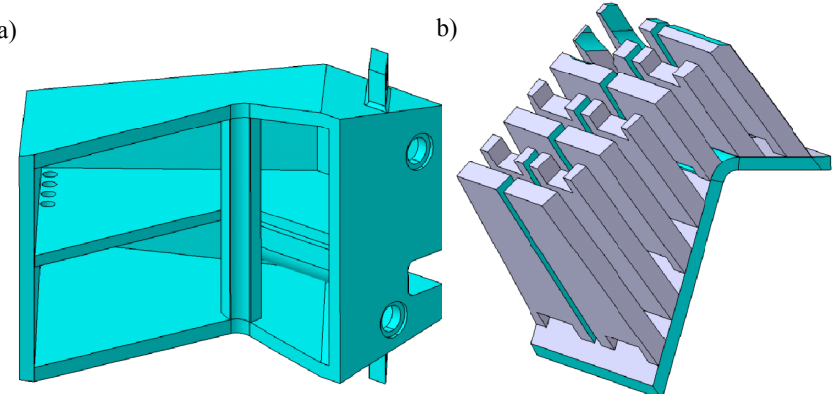

Figure 14. The (a) cask and (b) steel plate stacks which form the BSM top-level shield block based on the encased shield block concept.

adaptation of the plate and interspace dimensions, the optimum SS to water concentration (80/20 vol. \%) for the neutron shielding in the high flux area can be readily achieved [35].

\section{EM analysis}

The UL design has to withstand relatively large electro-magnetic (EM) and thermal-mechnical loading due to its vicinity with the ITER plasma and the transmission of high power microwave beams. This chapter is devoted to issues associated with the EM loading, while the following chapter discusses the thermal-mechnical aspects. During a plasma VDE, the current (up to 17MA) is rapidly quenched resulting in a changing poloidal magnetic field, which will induce potentially large currents in conducting materials. These induced currents then interact with the background toroidal and poloidal magnetic field to generate torques on all current conducting components. The magnitude of the torque depends on the component size, resistivity and the poloidal flux intercepting the components cross sectional area. Two components that are particularly susceptible to relatively large EM forces are the port plug structure and the steering mirror assembly.

To analyse the associate EM forces on these components, a three step process was adpoted. First, the transient magnetic field is estimated in the vicinity of the port plug arising from a VDE. Note that of the three disruptions types, ITER-IO determined that the VDE was determined to generate the largest transient fields in the vicinity of the UL. The quench of the plasma current is assumed to decay linearly within 36ms [38], further analysis assuming exponential decay is under progress. Next the induced current in a given component (port plug, steering mirror, shield block, etc.) is calculated based on the previously calculated transient magnetic field. Then the induced EM torques are estimated from the Lorentz force between the induced current and the background toroidal and poloidal magnetic fields.

The transient magnetic field in the vicinity of the upper port during a VDE was modelled in ANSYS ${ }^{\mathbb{R}}$ using a $20^{\circ}$ segment of the torus assuming toroidal symetry. The ANSYS ${ }^{\circledR}$ model as shown in figure 15 consists of three types of elements: vacuum (red), conductive (light blue) and the boundary surface (dark blue). Virtual vacuum loops represent the poloidal field coils forming the static magnetic field. During the VDE a current is induced to the plasma facing modules. This transient current distribution is projected on a cell layer artificially set to conductive vacuum directly in front of the blankets resulting in the fast changing poloidal field affecting the outer components. This resulting poloidal field variation is then used to estimate the induced currents in the launcher components (in particular the port plug and steering mirror). 


\section{M.A. Henderson et al}
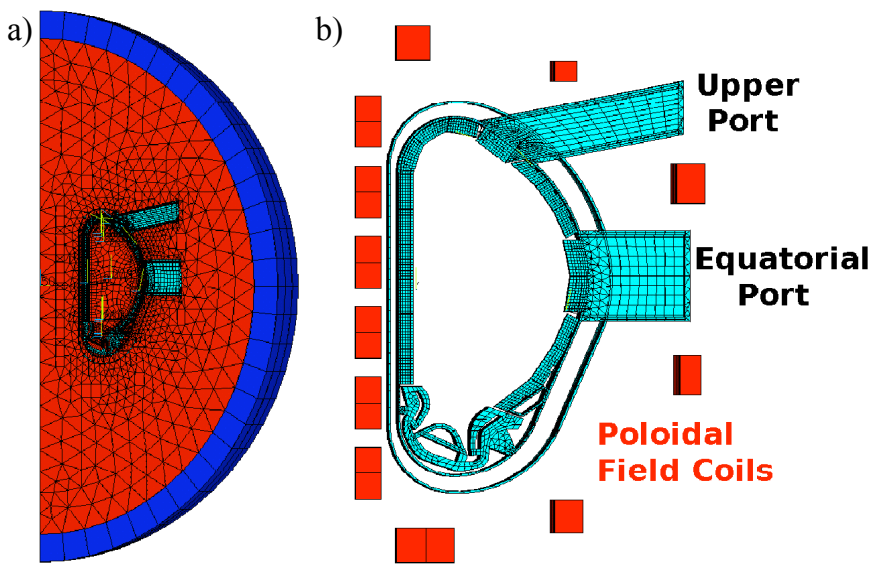

Figure 15. The torus segment and the modeled components.
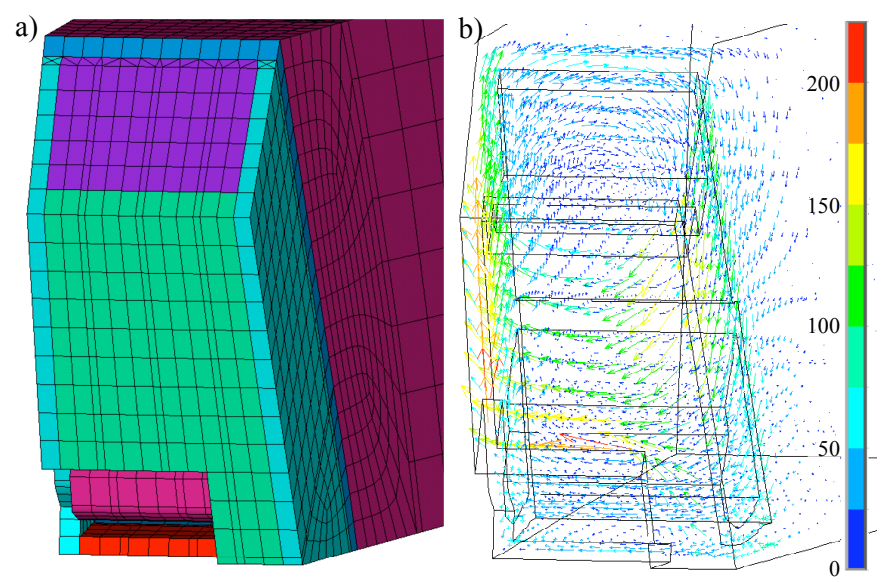

Figure 16. a) A simplified model of the port plug structure used for the EM analysis and b) the resulting induced current density vectors (color gradient in $M A / m^{3}$ ) in the BSM volume during a VDE.

\subsection{EM analysis of the port plug structure}

For the port plug, the transient poloidal field is applied to a simplified model of the launcher imported from a CATIA model as shown in figures 16a. The launcher is isolated from the torus by a small vacuum gap except at the launcher back end flange where the launcher is fixed to the vacuum vessel, which is assumed to be a rigid connection. The BSM volume includes shield blocks, focusing and steering mirrors and an asymmetric cut out in the first wall panel for the mm-wave beam passage. The internal shield section is filled with the two shield blocks in the front and middle sections with the rear part kept empty (see description of shield blocks in section 2.3.). Inside the upper port the simulation calculates the formation of eddy currents in the simplified port plug structure, as shown in figure 16b. These currents are then mapped to a more detailed port plug structure based on the CATIA ${ }^{\circledR}$ model of figure 2 imported into ANSYS ${ }^{\circledR}$ workbench 11.0 in the form of a meshed model. This procedure provides a relatively accurate characterisation of the induced currents in the BSM and port plug structure and a more conservative estimate for the internal shielding components.

During post processing the strong toroidal field is added to calculate the Lorentz force on each element of the port plug ANSYS ${ }^{\circledR}$ model. Force application points are created within the geometric center of each component and associated with the summed up force and a torque moment, see table 3 . The segment border between BSM 


\section{Article: Overview of the ITER EC UL}

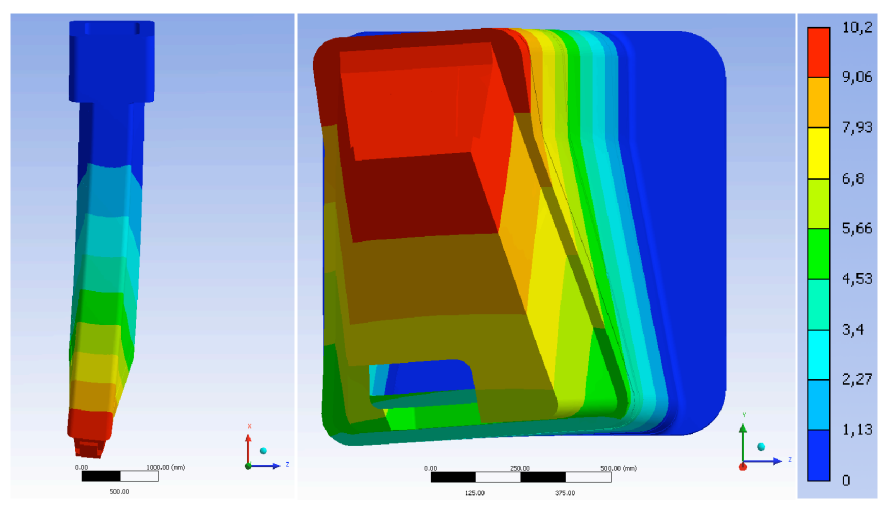

Figure 17. Axial bending and tilting of the launcher.

and internal shield cuts the major current loop in two parts causing a sign flip in the radial and poloidal force components.

Table 3. Forces and loads on different launcher components relative to their geometric center.

\begin{tabular}{|r|c|c|c|c|}
\hline Component vs. loads & BSM & Internal Shield & Middle & Rear \\
\hline$F_{\text {rad }}(\mathrm{MN})$ & -0.1 & 0.07 & 0.15 & 0.01 \\
\hline$F_{\text {pol }}(\mathrm{MN})$ & 0.2 & -0.17 & 0.03 & 0.0 \\
\hline$F_{\text {tor }}(\mathrm{MN})$ & -0.08 & -0.12 & -0.03 & 0.0 \\
\hline$M_{\text {rad }}(\mathrm{MNm})$ & -0.45 & -0.7 & -0.32 & -0.02 \\
\hline$M_{\text {pol }}(\mathrm{MNm})$ & -0.7 & -0.7 & -0.17 & -0.01 \\
\hline$M_{\text {tor }}(\mathrm{MNm})$ & 0.0 & 0.05 & 0.0 & 0.0 \\
\hline
\end{tabular}

In the present design stage the internal components such as shield blocks and their fixation are still subject to change. Optimisation of the volume, ratio between steel and water, and the fixation system are still under progress and will result in lower induced currents. The present un-optimised model was used as a first approach to calculate the EM load distribution and to evaluate a static elastic analysis of the launcher deformation. In this approximation, the fixation at the back end to the vacuum vessel is assumed to be held rigidly. The upper launcher is especially sensitive to bending in toroidal direction and tilting around the launcher main axis due to its trapazoid cross section, as illustrated in figure 17. The resulting bending of the port plug is listed in table 4 based on the calculated torques of table 3 . The most critical bending $(\sim 8 \mathrm{~mm})$, which occurs in the toroidal direction is mainly caused by $F_{\text {tor }}$ and $M_{\text {pol }}$. An additional tilting by $M_{\text {rad }}$ increases the toroidal launcher deformation to $10.2 \mathrm{~mm}$. The maximum spacing to neighboring components is $20 \mathrm{~mm}$ leaving slightly less than $10 \mathrm{~mm}$ tolerance for compensating the inaccuracy associated with installing the port plug relative to the vacuum chamber, which have yet to be determined.

Note that these results provide an estimate on the present design and aid in estimating how large the forces and resulting deformations are during a VDE. Further fine tuning of the model is required that includes adding the shield blocks into the ANSYS model of figure 17, which will influence the port plug inertia and spring constant.

The above deformations induce high stresses that are concentrated at the transition from the trapezoid structure at the closure plate to the stiffer launcher back end. The stress maximums occur on the upper two corners of the trapezoid reaching $200 \mathrm{MPa}$ (see figure 18) compared to $S_{y}\left(125^{\circ} \mathrm{C}\right)=210 \mathrm{MPa}$, which is the limit for onset of high cycle fatigue. As the simulation was performed static these values can only be taken as a 


\section{M.A. Henderson et al}

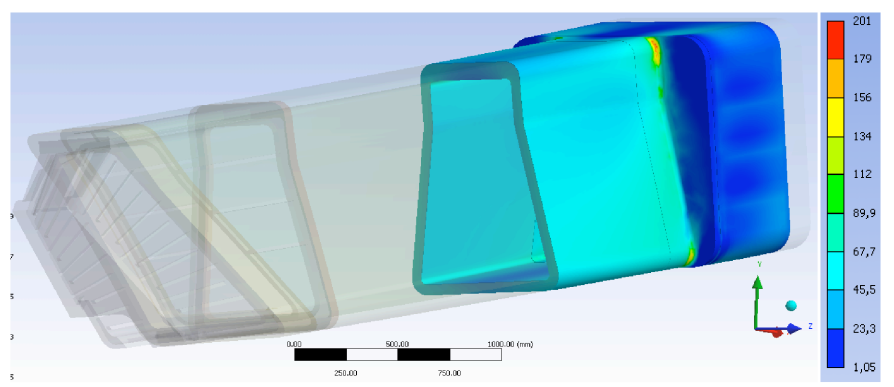

Figure 18. Von Mises stresses $[\mathrm{MPa}]$ under differential loads in the rear part are significant for the deformation at the free launcher tip.

first approximation of induced stresses. However the stress peak is in a range tolerable to level $\mathrm{C}$ events (an emergency event defined in the ITER structural design criteria for in-vessel components), where local permanent deformations are allowed as long as immediate fracture can be excluded with reasonable confidence.

Table 4. Launcher deformation under difference loads. The differential loads arise from table 3, the BSM and internal shield loads are taken from previous analysis.

\begin{tabular}{|r|c|c|c|}
\hline Deformations $[\mathrm{mm}]$ & Differential & BSM + Int. shield & UPP loads \\
\hline Radial & 1.2 & 1.3 & 1.8 \\
\hline Poloidal & 0.8 & 0.7 & 2.5 \\
\hline Toroidal & -10.2 & -11.4 & -11.8 \\
\hline
\end{tabular}

These EM load calculations are 10\% lower than the EMAS [39] calculations and 15\% lower than the older reference loads. Although the estimated stresses are very close to the allowable limit for the $15 \mathrm{MA}$ plasma case, further design improvements associated with the attachment of the trapezoidal structure to the closure plate could significantly reduce the peak stress levels. The present configuration assumed a simple attachment of the main structure to the closure plate, without optimising the connection. The transient analysis taking into account the time varying poloidal field and the more critical case of a 17MA plasma current (scenario 5) will be performed once the fixation of the internal shield blocks has been finalised. Recall, the goal of the exercise was to determine where the maximum stresses occurred for further optimisation, which will occur in the future port plug design.

\subsection{EM analysis of the steering mirror}

The fast poloidal field variation estimated above is also used for estimating the induced currents in the steering mirror assembly, but scaled for a 17MA plasma current. For the steering mirror assembly there are several concerns associated with the EM loading, which include:

- induced currents in the steering mirror

- halo currents flowing through the port plug and steering mirror structures

- induced currents in the spiral cooling pipes

- torque on the steering mirror causing it to rotate during a VDE

- induced torque resisting rotation of the steering mirror 


\section{Article: Overview of the ITER EC UL}

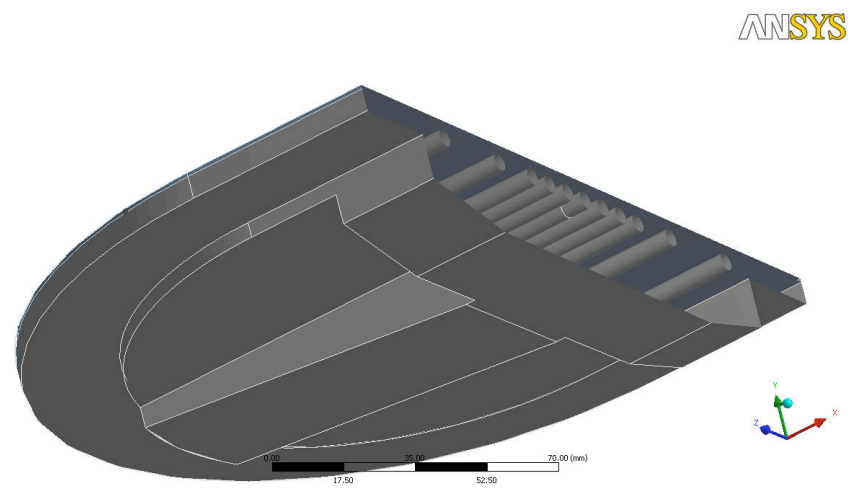

Figure 19. Cross sectional view of the steering mirror design with a distributed cooling channel for minimising the surface thermal gradients.

Of these issues the most critical was the induced current in the steering mirror during a VDE, which is described in more detail below. All the other issues could be resolved by introducing voltage isolation breaks or resulted in relatively small torques that were not critical to the operation or reliability of the steering mirror assembly [24].

The choice of the steering mirror configurations (see figures 7 and 9) depend on the amount of induced current and resulting torque in the steering mirror during a VDE. A cut away view of the steering mirror is shown in figure 19 based on an ANSYS model used to simulate the thermal, mechanical and EM properties of the mirror. The optimisation procedure aims at reducing the total mass around the mirror edges, which minimises the amount of current that circulates in the mirror during a VDE and reduces the inertia for faster rotation. The mirror is formed from a thin copper sheet supported by a stainless steel back with cooling channels included in the body. The coolant channel spacing is chosen for optimal evacuation of the thermal loading $\left(\sim 2 M W / m^{2}\right.$ assuming four 2MW incident beams) and minimising the thermal deformation.

The estimated torque arising from the VDE assumed a time varying magnetic field of $25 T / s$ based on the estimations from the torus model of figure 15 and a plasma current of 17MA. The mirror model was simplified (cooling channels filled in) to reduce the calculation time and provide a pessimistic estimation. At present the induced torque on the mirror is $\sim 300 \mathrm{Nm}$ at the worst rotation angle relative to the poloidal magnetic field. This torque generates a force of $\sim 1.5 k N$ on the flexure pivots, which (as mentioned above) have been designed to support up to 1'000 VDEs of $3.2 k N$ far exceeding the estimated 100 VDEs on ITER. The steering mirror design is still undergoing further optimisation attempting to reduce the induced current and rotational inertia for additional safety margin of the mechanical system. A first prototype mirror design for thermal-mechanical testing is planned for the end of 2007.

\section{Thermo-Mechanical analyses}

The UL components will be heated from two sources: the RF beam incident on the mirror surfaces and the thermal and nuclear radiation from the plasma. The thermal loading associated with the RF beam creates the highest thermal loading in the UL, which is predominately on the mirror surfaces with peak power densities $\leq 4 \mathrm{MW} / \mathrm{m}^{2}$ assuming $2 \mathrm{MW}$ per beam. Replacing the mitre bends with free space mirrors reduces the peak power density occuring in the optical systems to $\leq 3 \mathrm{MW} / \mathrm{m}^{2}$, which would simplify the mirror design and increase the engineering safety margin. As explained in section 2.2., the next design envisions to avoid the use 


\section{M.A. Henderson et al}

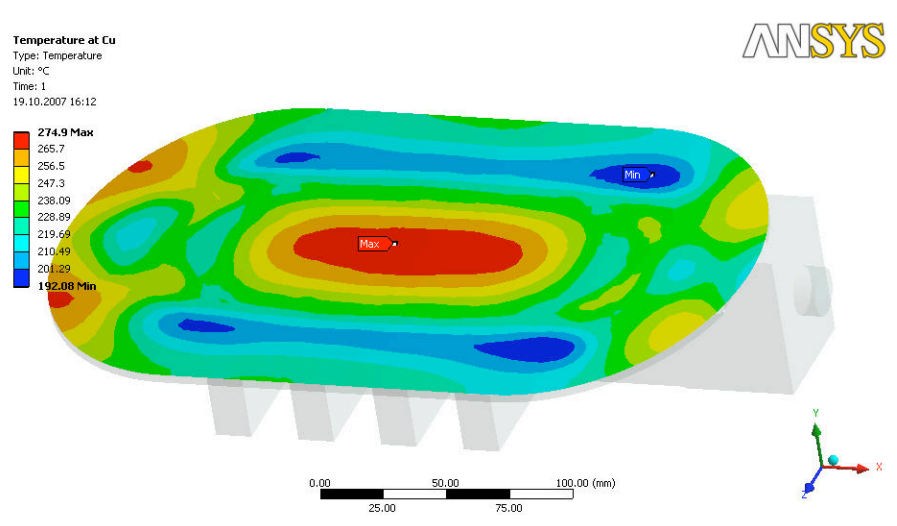

Figure 20. The thermal profile of the steering mirror assuming four $2 \mathrm{MW}$ incident beams.

of the mitre bends, which will also improve the $\eta_{N T M}$ efficiencies, reduce the overall costs and simplifies the optical system. As has been shown in reference [24], power densities in the range of $\leq 4 M \mathrm{~W} / \mathrm{m}^{2}$ can be achieved by conventional cooling techniques, so the coolant of the RF components is not a critical issue for either optical configuration.

Most of the engineering effort associated with the thermo-mechnical analysis of the mirrors has concentrated on the design of the steering mirror, which has to have a high thermal conductive centre to remove the deposited heat load, while avoiding a large volume of highly electrical conductive material to minimise the induced currents during a VDE. In addition, ITER-IO recommends that the cooling channels do not have direct contact with copper. The present mirror design envisions a SS body (low electrical resistivity) with imbedded cooling channels machined into the body and then capped by welding a $0.7 \mathrm{~mm}$ thick SS sheet on top. A copper coating is electro deposited on the surface of variable thickness (thin at the edges to minimse the induced currents) and polished for a near optical surface finish. The $0.7 \mathrm{~mm}$ thick SS insures the mirror can sustain proof pressure testing of up to 62.5 bar. The ANSYS mirror design of figure 19 has been analsyed as shown in figure 20 with a peak central temperature of less than $280^{\circ} \mathrm{C}$ and maximum induced stress intensity of $\sim 300 \mathrm{MPa}$ located in the copper at the SS interface near the peak temperature. Note that this satisfies the ITER guidelines for copper and SS and assuming input cooling temperatures of $115^{\circ} \mathrm{C}$. The mirror surface was assumed pure copper with a surface roughness factor of 2.0 and a resistivity of $1.724 e^{-8} \Omega m$ (copper at $280^{\circ}$ ). The designs of the other mirrors forming the mm-wave optical system have not been advanced beyond a conceptual design study. Once a reliable mirror design is obtained for the steering mirror, it will be used for the other mirrors that have less restrictive design requirements.

The thermal and nuclear radiation is roughly an order of magnitude lower than the RF, estimated at $\sim$ $0.2 \mathrm{MW} / \mathrm{m}^{2}$ (thermal radiation for internal components visible to the plasma) and $\leq 1.5 \mathrm{MW} / \mathrm{m}^{3}$ (nuclear radiation depending on material and location in the port plug). These later heating sources have a negligible impact on the optical mirrors, but a significant impact on components that have poor thermal conductivity and long conductive path lengths to coolant channels for example the bellows used in the pneumatic system of the steering mirror. This can be overcome by utilising a combination of radiative and conductive cooling. The housing surrounding the bellows will be actively cooled and kept at $\leq 120^{\circ} \mathrm{C}$, the absorbed power in the bellows wall will be both conducted and radiated to this surface resulting in a peak bellows temperature of $\leq 190^{\circ} \mathrm{C}$ [24].

The thermal loading in the port plug structure, BSM and shield blocks is cooled using the blanket cooling water circuit, which is also used for torus baking. ITER requires the components exposed to the torus vacuum 


\section{Article: Overview of the ITER EC UL}

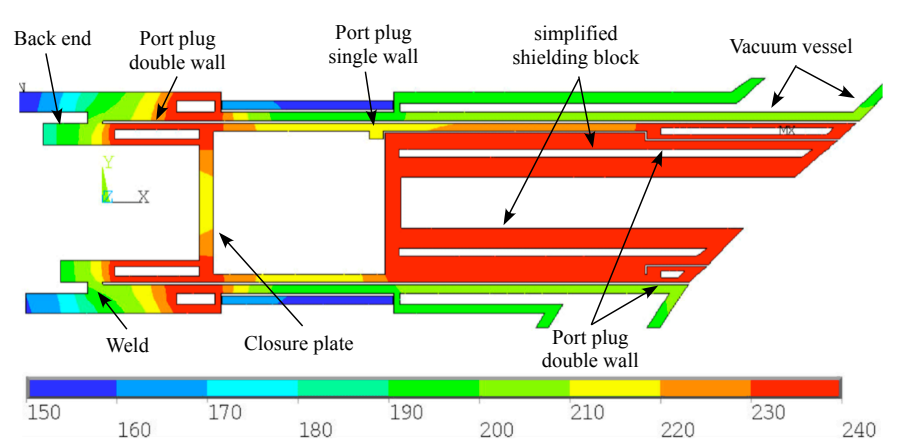

Figure 21. Design base for the temperature analysis for the baking process of the port plug in the surrounding vacuum vessel and port extension (top) and the profile calculated for the temperature distribution attained with a baking period of $100 \mathrm{~h}$ (bottom).

to be baked at $\leq 240^{\circ} \mathrm{C}$. Since in the present slim wall port plug design, there are long paths in stainless steel for the heat to flow in the central single wall section, the radiation heat transfer among components and to the environment will play a major role in the baking process.

The temperature analysis of the port plug system was performed by $2 \mathrm{D}$ finite element modeling using the ANSYS simulation tools including the heat loss due to radiation. The model, as given in figure 21, simulates the vertical mid plane of the launcher, of the vacuum vessel port and of the port extension. The equilibrium of the temperature distribution could be found during the prescribed baking period of 100 hours with blanket water at $240^{\circ} \mathrm{C}$ following a temperature ramp-up of $50 \mathrm{~h}$. At temperature equilibrium, all parts of the port plug attained temperatures above $200^{\circ} \mathrm{C}$ which is expected to give a sufficient margin to the minimum baking temperature of SS surfaces of $180^{\circ} \mathrm{C}$

\section{Summary}

The ITER EC upper port launcher is nearing completion of the detailed design and will soon be progressing toward the final build to print design scheduled for completion in 2009. The design work has been described based on the three principle subsystems comprising of the launcher: port plug, mm-wave assembly and shield blocks. The present port plug design provides a rigid structure capable of withstanding the EM forces, while providing a relatively thin wall to insure adequate space for the mm-wave components. The wall has a varying thickness along its length with a double wall structure near the plasma to provide cooling to the structure during operation and a thin wall in most of the body that reduces manufacturing costs. A hybrid port plug designs is under investigation that provide increased access to the internal launcher components using a hatch positioned on top of the port plug.

The mm-wave system has been designed to provide the narrowest deposition across a majority of the plasma cross section. The design has been modified to increase the overall access range in the plasma to access the $q=1$ surface for the control of the sawtooth instability and to relax the steering requirements of the equatorial launcher. Further enhancements of the optical design are under progress that consider the replacing the mitre bends with free space mirrors. This design modification reduces the overall cost and complexity of the mm-wave components while reducing the astigmatism of the injected beam toward the plasma for improved performance in controlling MHD activity. The launcher achieves its principle goal of stabilising the NTMs with a relatively large margin of safety ranging from a factor of 1.8 to 3.5 relative to the goal of $\eta_{N T M}=1.2$. The critical mm- 


\section{M.A. Henderson et al}

wave component is the steering mirror assembly, which has all critical issues resolved and is now progressing toward the manufacturing of an ITER-like prototype design planned for testing in mid 2009.

Preliminary design of the shield blocks that fill in the remaining volume between the mm-wave beams and the port plug structure has been described. The shield blocks are used both in the BSM volume and in the main structure. Three different designs have been investigated with the optimum chosen depending on the location in the port plug, size and shape. The thermo-mechanical analysis of some of the critical components have been presented including the steering mirror and port plug structure.

The electro-magnetic induced forces arising from a VDE have been described for both the steering mirror and the port plug structure. The calculated induced currents in the port plug structure are within range of limiting the internal stresses sufficiently below the ITER design specifications upon further optimisation. The forces arising from the induced currents in the steering mirror assembly are sufficiently low such that the steering mirror assembly can withstand up to 1'000 VDE's (17MA) at the worst mirror rotation, which is an order of magnitude more than required by ITER. In addition, the thermal loading of the steering mirror has been estimated using finite element models. The maximum temperature and stresses are acceptable for the copper and SS forming the mirror body.

In conclusion, the design and analyses of the UL is on track with the ITER schedule aiming for a first plasma in 2016. With all critical issues having a solution that is now at or near the detailed design level.

\section{Acknowledgments}

This work, supported by the Swiss National Science Foundation and the European Communities, was carried out within the framework of the European Fusion Development Agreement (ECHULB subtasks (a), (b) and (f) / contract EFDA 05-1228). The views and opinions expressed herein do not necessarily reflect those of the European Commission.

\section{References}

[1] G. Saibene et al, Design of the ITER Electron Cyclotron Wave launcher for NTM Control, in Fusion Energy 2006 (Proc. 21st Int. Conf. Chengdu, 2006) (Vienna: IAEA) CD-ROM file IT/P2-14 and http://wwwnaweb.iaea.org/napc/physics/FEC/FEC2006/html/index.htm.

[2] M.A. Henderson et al, Fusion Eng. Design 82454 (2007).

[3] K. Takahashi et al Fus. Science and Tech. 471 (2005).

[4] K. Takahashi et al, Journal of Physics: Conference Series 2575 (2005).

[5] S. Gunter et al, Phys. Rev. Lett. 87 275001-1 (2001).

[6] C. Angioni et al, Nucl. Fusion 43455 (2003).

[7] R.J. La Haye et al, Nucl Fus 46451 (2006).

[8] H. Zohm et al, Journal of Physics: Conference Series 25234 (2005).

[9] G. Ramponi et al, Capabilities of the ITER ECRH/ECCD Systems for Extended Physics, Proc. of the $14^{\text {th }}$ Joint Workshop on ECE and ECRH, Santorini, Greece (2006) and http://www.hellasfusion.gr/englishver/allcontribut.php.

[10] E. Poli et al, Performance Studies for the ITER ECRH Laucnher, Proc. of the $14^{\text {th }}$ Joint Workshop on ECE and ECRH, Santorini, Greece (2006) and http://www.hellasfusion.gr/englishver/allcontribut.php. 


\section{Article: Overview of the ITER EC UL}

[11] M.A. Henderson et al, Synergy Study of Eq. and Upper Port Launchers for an enhanced Physics Performance, Proc. of the $14^{\text {th }}$ Joint Workshop on ECE and ECRH, Santorini, Greece (2006) and http://www.hellasfusion.gr/englishver/allcontribut.php.

[12] G. Ramponi et al, Fusion Science Tech. 52193 (2007).

[13] J. Graves et al, Plasma Phys. Control. Fusion 47 B121 (2005).

[14] Project Integration Document ITER_D_2234RH V. 3.0 (2007).

[15] R. Heidinger et al, Journal of Physics: Conference Series 2566 (2005).

[16] R. Heidinger et al, Structural system of the ECH Upper Port Plug for ITER, Proceedings $4^{\text {th }}$ IAEA ECRH Technical Meeting, May 2007, Vienna, Austria.

[17] R. Heidinger et al, Design and Analysis of the ECH Upper Port plug Structure at ITER, in Fusion Energy 2006 (Proc. 21st Int. Conf. Chengdu, 2006) (Vienna: IAEA) CD-ROM file IT/P2-10 and http://wwwnaweb.iaea.org/napc/physics/FEC/FEC2006/html/index.htm.

[18] P.Spaeh et al, Fusion Eng. Design, 82700 (2007).

[19] L. Doceul et al, Fusion Eng. Design 821216 (2007).

[20] E. Ciattaglia et al, Fusion Eng. Design 821231 (2007).

[21] P. Sherlock et al, Fusion Eng. Design, 821806 (2007).

[22] G. Ramponi et al, Physics analysis of the ITER ECW system for an optimized performance, submitted to this issue of Nuclear Fusion.

[23] H. Shidara et al, ECRH Beam optics optimization for ITER Upper Port Launcher, Proc. of the $14^{\text {th }}$ Joint Workshop on ECE and ECRH, Santorini, Greece (2006) and http://www.hellasfusion.gr/englishver/allcontribut.php.

[24] M.A. Henderson et al, Critical Design Issues of the ITER ECH FS Upper Launcher, to be published in Fusion Science and Technology January, 2008.

[25] R. Chavan et al, Fusion Eng. Design 82867 (2007).

[26] R. Chavan et al, Journal of Physics: Conference Series 25151 (2005).

[27] J.-D. Landis et al, Fusion Eng. Design 82897 (2007).

[28] H. Grunloh et al, A steerable ECRF launcher for ITER, Proceedings of the Twentieth Symposium on Fusion Technology, Marseille, September, 1998.

[29] M.A. Henderson et al, Critical Interface Issues associated with the ITER EC System, submitted to this issue of Nuclear Fusion.

[30] M.A. Henderson et al, Fusion Eng. Design 53 (2001) 241.

[31] Expansion Joint Manufacturers Association, http://www.ejma.org/.

[32] R. Heidinger et al, Fusion Eng. Design 82693 (2007).

[33] D. Rasmussen et al, Design of the ITER Electron Heating and Current Drive Waveguide Transmission Line, Proceedings $4^{\text {th }}$ IAEA ECRH Technical Meeting, May 2007, Vienna, Austria.

[34] M.A. Henderson et al, Nucl. Fusion 251487 (2004).

[35] A. Serikov et al, Fusion Eng. Design 82736 (2007).

[36] A. Serikov et al, Nuclear Analyses for the ITER ECRH Launcher, submitted to this issue of Nuclear Fusion.

[37] N. Miki et al, Fusion Eng. Design 58-59 (2001).

[38] M. Sugihara et al, Nucl. Fusion 47 (2007) 337.

[39] EMAS (Electric and Magnetic Analysis Software) licensed from the MacNeal- Schwendler Corp. 